Angiboust, S. (2014). La parole aux images, ou Multilinguisme et traduction dans les films de John McTiernan. Linguistica Antverpiensia, New Series. Themes in Translation Studies, 13, 14 29.

\title{
La parole aux images, ou Multilinguisme et traduction dans les films de John McTiernan
}

\section{Sylvain Angiboust}

Chercheur indépendant

sylvainangiboust@gmail.com

Most of modern Hollywood action movies are Manichean and ethnocentric but John McTiernan's films are surprisingly scrupulous in the depiction of other cultures and the use of foreign languages. The director first uses theses languages for their musicality and exotic feeling. The ability to speak is depicted as the main characteristic of humanity. The understanding of the other one is based on the knowledge of its language, so the interpreter became a very important role in McTiernan's movies. The interpreter's character appears as a link between the individuals, wrangled over their own language and culture. The interpreter is also standing for the spectator and leads him into unknown worlds. John McTiernan does not only make movies about interpreters. The act of translation appears to be the one of the most important aspects of his cinematographic style. McTiernan considers spoken words mostly as noises and thinks that the real meaning of the movie is delivered by the pictures themselves: the expressivity of cinema is the one of framing and camera moves. The audience may not understand the language spoken by the characters but he can always rely on the pictures, which are meaningful. McTiernan's mise en scene becomes a substitute for spoken language.

\section{Introduction}

Plusieurs héros de films d'action américains des années 1980-90 sont d'origine étrangère. L'Autrichien Arnold Schwarzenegger (Terminator [The Terminator, James Cameron, 1984], True Lies [J. Cameron, 1994]), le Belge Jean-Claude Van Damme (Bloodsport [Newt Arnold, 1988], Universal Soldiers [Roland Emmerich, 1992]) et l'Italo-Américain Sylvester Stallone (Rocky [John G. Avildsen, 1976], Rambo [First Blood, Ted Kotcheff, 1982]) ont été choisis par le public du monde entier - qui fit un triomphe à leurs films - pour incarner un idéal fantasmé d'héroïsme et de virilité américaine, voire une forme de nationalisme. ${ }^{1}$ 
Des générations entières d'acteurs se sont vus contraints d'angliciser leur nom sous peine de passer à côté de la célébrité. Schwarzi, lui, arbore fièrement le patronyme le plus imprononçable et le plus alambiqué jamais imprimé sur l'affiche d'un film américain. (Hoberman, 1991, p. 51)

Ces stars ne cachent pas leurs origines : Rocky Balboa est un héros du melting pot, les personnages de Van Damme portent des noms «vieille France » (Léon Gaultier, Luc Deveraux, Jean Vilain...) et Schwarzenegger interprète d'abord des personnages étranges (le robot de Terminator) ou étrangers (un policier russe dans Double détente [Red Heat, Walter Hill, 1988]). Le cinéma hollywoodien a intégré l'altérité de ces stars exotiques, les a «traduites » en les adaptant à un contexte américain où elles sont opposées, de façon manichéenne, à des adversaires qui, eux, parlent une autre langue, inconnue de la plupart des spectateurs et présentée comme une menace. Ainsi, les militaires qui s'en prennent au héros de Rambo 2 : la mission [Rambo: First Blood Part II, George Pan Cosmatos, 1985] parlent vietnamien et russe, les trafiquants de drogue sud-africains de L'Arme fatale 2 [Lethal Weapon 2, R. Donner, 1989] s'expriment en afrikaans et les terroristes de True Lies en arabe.

Le réalisateur John McTiernan a dirigé à deux reprises Schwarzenegger (dans Predator et Last Action Hero) ${ }^{2}$, mais il est surtout à l'origine du héros le plus typiquement américain de la période, le policier new-yorkais John McClane (interprété par Bruce Willis, né de mère allemande mais élevé aux États-Unis), cow-boy moderne opposé à des terroristes est-allemands dans Piège de cristal et sa suite Une journée en enfer. Dans ce diptyque, les personnages communiquent constamment par radio ou téléphones interposés, et sont amenés, comme dans les autres films de McTiernan, à parler des étrangères.

Nous avons choisi de nous intéresser à l'œuvre de McTiernan à la fois pour son exemplarité (ses films constituent le mètre-étalon du cinéma pyrotechnique et musclé) et sa singularité : nous allons en effet voir que le cinéaste porte une attention toute particulière au langage et aux différentes formes de communication. Il s'intéresse en particulier au processus de traduction, grâce auquel un héros anglophone peut acquérir la maîtrise d'un environnement géographiquement et culturellement étranger (À la poursuite d'Octobre rouge, Le Treizième guerrier). Au sein $\mathrm{du}$ genre physique du film d'action, que peut apporter le processus intellectuel de la traduction ? Les films de McTiernan nous permettent de réfléchir à l'utilisation des langues étrangères par un cinéma de divertissement hollywoodien qui n'a rien d' «aveuglément monoglotte » («blindly monoglot» Cronin, 2008, p. xiii). Le cinéaste a d'ailleurs pour habitude de renverser dans ses films certains des stéréotypes en vigueur dans le reste de la production hollywoodienne : son film de Guerre froide À la poursuite d'Octobre rouge a pour héros un Soviétique (interprété par l'acteur écossais Sean Connery) et le spectateur du Treizième guerrier est 
invité à s'identifier à un Arabe (interprété par l'Espagnol Antonio Banderas). La bande-son des films rend compte de la diversité des langues parlées mais c'est l'image qui est pour McTiernan le principal vecteur d'expressivité. Le réalisateur conçoit le cinéma comme un médium principalement visuel, où la signification n'est pas prise en charge par les mots (dont le sens est culturellement contingent) mais par un enchaînement d'images en mouvement. Il voit là une langue universelle, capable de traduire le multilinguisme des sociétés humaines dans un idiome visuel commun; une proposition que cet article se propose d'interroger sous l'angle de la science de la traduction.

Le multilinguisme est dans un premier temps utilisé par le cinéma d'action hollywoodien contemporain pour mettre en valeur, par contraste, la langue anglaise du héros. Les films de John McTiernan s'écartent de ce manichéisme en faisant du processus de traduction à la fois un moteur narratif et un principe esthétique. Il apparait enfin que le réalisateur envisage les images en mouvement comme un équivalent, voire un substitut à la communication verbale: il se propose de traduire visuellement le contenu sémantique des dialogues.

\section{Multilinguisme et altérité}

On parle peu dans les films d'action. Stallone ou Schwarzenegger sont plus célèbres pour leurs grognements et leurs cris (Rocky hurle le nom de sa fiancée au terme d'un combat de boxe) que pour leurs dialogues. Le multilinguisme est pourtant bien présent dans les films d'action, et de façon parfois importante : le premier quart d'heure de Double détente est intégralement en russe sous-titré, les dialogues passant à l'anglais lorsque l'intrigue se déplace à Chicago. Ivan Drago, le gigantesque boxeur soviétique qu'affronte Stallone dans Rocky IV [Sylvester Stallone, 1985], parle peu et les Américains se moquent de lui en disant que «sa langue a été saisie à la douane» («The man's tongue didn't come through custom. ») : derrière la boutade se trouve une remarque pertinente sur la situation de l'individu en terre étrangère. Drago a besoin d'un traducteur, son épouse s'exprime à sa place dans un anglais parfait : il est le corps, elle est l'esprit et la voix. Les rares répliques du boxeur se font dans un anglais hésitant, d'une concision aussi brutale que ses coups de poing : «Tu vas perdre » («You will lose »), «Personne ne peut me battre » («I cannot be defeated $»$ ). Partagé entre un silence déshumanisant et une parole brute et maladroite, Drago est comparable à l'Amérindindien du cinéma classique, qui était «d'un silence menaçant ou alors vociférait violemment. » (« The 'Indians' were bogeymen, either menacingly silent or vociferously violent. » Cronin, 2008, p. 46) L'altérité langagière se double d'un jugement moral : dans ces films, celui qui ne parle pas (ou parle mal) anglais est, jusqu'à preuve du contraire, considéré comme une menace. 
La représentation du mode de vie américain dans les films populaires est très artificielle :

Tout se passe comme si la production cinématographique que Hollywood exporte un peu partout avait fini par créer une perception stéréotypée de l'Amérique, qui est alors susceptible d'accueillir sans effort des films qui alimentent et renforcent ces stéréotypes. (Amiel et Couté, 2003, p. 120)

Le même traitement stéréotypé est réservé aux pays étrangers (les cartes postales ensoleillées que traverse James Bond), à leur langue ainsi qu'à leur musique (chants slaves lorsqu'un personnage est russe, percussions arabisantes dès que l'on se trouve dans le désert). Ce schématisme est encore plus marqué lorsqu'il touche aux pays «ennemis » des États-Unis. On pourrait établir une cartographie des tensions politiques américaines à partir des langues parlées par les méchants de films d'action : le russe des années 1980, témoignage de la Guerre Froide, est désormais remplacé par le coréen de l'Axe du Mal (Meurs un autre jour [Die Another Day, Lee Tamahori, 2002], La Chute de la Maison Blanche [Olympus Has Fallen, Antoine Fuqua, 2013]). Ce genre de films « est un commentaire précis de la production américaine de stratégie, à mesure que s'opère celle-ci. » (Valantin, 2010, p. 7)

La façon dont ces méchants parlent leur langue nous en apprend sur leur hiérarchie. Les ennemis «figurants» - hordes de soldats indifférenciés qui tombent sous les balles du héros -parlent une seule langue, étrangère, réduite à quelques onomatopées. Les véritables adversaires du héros, militaires ou terroristes, ont, eux, droit à d'authentiques dialogues : ils parlent anglais avec un fort accent, mais n'oublient jamais d'insérer quelques mots dans leur langue maternelle. C'est un plurilinguisme de convention, expression générique de la «nonaméricanité » des personnages négatifs, renforcée par le fait que ces étrangers sont rarement interprétés par un acteur partageant leur origine : c'est le cas y compris chez McTiernan, où les Britanniques Alan Rickman et Jeremy Irons jouent des Allemands dans Piège de cristal et Une journée en enfer et le Français Jean Réno un oligarque russe dans Rollerball. En effet, «les acteurs venus d'Europe représentent des nationalités souvent interchangeables pour les casting directors. » (Vincendeau, 2009, p. 199)

L'allemand parlé par les preneurs d'otages de Piège de cristal est moins un effet de réel qu'une marque d'exotisme. Dès que le dialogue contient une information importante pour le spectateur, les Allemands se mettent automatiquement à parler anglais. Le besoin de traduction est un impératif extra-diégétique qui est intégré par les personnages : lorsque le criminel Hans Gruber donne l'ordre en allemand à son homme de main Karl de tirer sur des vitres pour blesser les pieds nus de John McClane, il le répète ensuite en anglais, de façon à être compris non seulement par le 
personnage germanophone mais aussi par le spectateur anglophone. Dans les versions doublées du film, la première réplique se fait en allemand avant d'être reprise dans la langue nationale ${ }^{3}$. L'allemand parlé par les preneurs d'otage est assez médiocre, parfois grammaticalement incorrect, et on est surpris d'entendre à la fin du film Hans Gruber dire à ses hommes de se dépêcher en français («Allez!»). Les répliques en langue étrangère de Piège de cristal ne sont donc pas à destination d'une minorité de spectateurs germanophones mais de la majorité de ceux qui n'identifient cette langue que par sa sonorité.

L'intérêt porté aux accents exotiques, à la musique des langues, est une constante chez McTiernan. Dans Nomads, son premier film, l'acteur irlandais Pierce Brosnan interprète un savant québécois que l'on entend parler français (ses répliques sont néanmoins à peu près incompréhensibles, à cause d'erreurs de syntaxe et de prononciation). Le décryptage de la langue étrangère est d'ailleurs le moteur de cette fiction. Au moment de mourir, le savant murmure à l'oreille de son infirmière une phrase mystérieuse que celle-ci devra traduire en anglais pour découvrir le secret de cette mort : "N'y sont pas ; sont des Inuats » (comprendre, dans un français correct : "Ils n'existent pas, ce sont des Inuats »). Dans Piège de cristal et Une journée en enfer, les frères Gruber, d'origine allemande, prennent des accents pour tromper leurs victimes: Hans se fait passer pour un Américain en imitant l'accent californien, alors que Simon fait semblant de bégayer puis parle anglais avec un accent hollandais de comédie.

Le multilinguisme des films de McTiernan est une forme de dépaysement. Ses héros anglophones sont plongés dans un monde verbalement hostile dont ils ne possèdent pas les codes: dans $L e$ Treizième guerrier, un Arabe voyage en compagnie de guerriers vikings et doit apprendre le vieux norrois pour se faire comprendre d'eux; dans Rollerball, un sportif américain participe à des compétitions violentes dans l'ancien bloc de l'Est. Retransmis dans le monde entier, les matchs sont commentés en direct par des présentateurs de toutes nationalités. McTiernan juxtapose leurs commentaires dans un brouhaha babélique dont l'absence de sous-titrage souligne la vacuité : l'issue truquée des matchs est connue à l'avance et les commentateurs disent tous la même chose, quelle que soit leur langue. La variété des langues ne correspond pas dans Rollerball à un idéal de société multiculturelle mais à sa version dégradée, la pensée unique d'un capitalisme mondialisé.

Chez McTiernan, comme dans les autres films d'action hollywoodiens, le héros est américain et/ou parle anglais. Son adversaire étranger, lui, est toujours au moins bilingue, maîtrisant à la fois sa langue maternelle et l'anglais. Ce polyglottisme nous permet-il de conclure à la supériorité intellectuelle du méchant sur le héros? Le méchant de film d'action est une figure du contrôle : il maîtrise l'espace (l'immeuble de Piège de cristal), les communications (Hans Gruber et ses hommes sont équipés de radios), ainsi que différentes langues. Dans Die Hard 4 : 
Retour en enfer [Live Free or Die Hard, Len Wiseman, 2004] quatrième aventure de John McClane à laquelle John McTiernan n'a pas collaboré - le méchant est pour la première fois un Américain, épaulé par des criminels étrangers (européens et asiatiques). Ce méchant donne ses ordres en anglais mais comprend également ses acolytes qui lui parlent en français et en italien : sa supériorité intellectuelle et tactique apparait dans sa façon de comprendre tous les personnages et d'être compris par eux.

Alors que le héros de Rollerball n'arrive pas à trouver sa place dans un monde multilingue saturé d'informations visuelles et sonores, le personnage principal du Treizième guerrier, Ahmed ibn Fadhlan, un poète arabe du $10^{\text {ème }}$ siècle en ambassade chez les Vikings, s'adapte à son nouvel environnement, en apprend la langue pour en comprendre la culture. Le manichéisme des films d'action hollywoodiens repose sur la négation de l'Autre, le stéréotypage des cultures : à l'opposé de cette tendance, l'œuvre de John McTiernan met l'accent sur les processus de traduction et d'apprentissage des langues.

\section{Parler d'autres langues}

Les films de McTiernan s'intéressent une forme d'altérité plus profonde que celle qui oppose deux hommes ne parlant pas la même langue. Le cinéaste questionne la nature humaine en opposant ses héros à du nonhumain (des fantômes dans Nomads et un extra-terrestre dans Predator, ses deux premiers films) ou à une pré-humanité (les Vikings du Treizième guerrier sont menacés par une tribu préhistorique en retard sur l'évolution). Cette opposition a des conséquences dans le domaine de la communication : le Prédator et la tribu des Wendols ne possèdent pas de langage articulé, ce qui confirme l'inhumanité de leur apparence physique. L'humain est le seul être vivant doté de la parole.

On a dit, non sans raison, que les sociétés primitives fixent les frontières de l'humanité aux limites du groupe tribal, en dehors duquel elles ne perçoivent plus que des étrangers, c'est-à-dire des sous-hommes, sales et grossiers, sinon des non-hommes : des bêtes dangereuses ou fantômes. (Lévi-Strauss, 1962, p. 220)

Le barbare est, étymologiquement, celui qui ne parle pas la langue. L'apprentissage d'une nouvelle langue et la traduction permettent de repousser les limites de cette «barbarie» en intégrant une part de l'altérité de son voisin et en le rejoignant dans un groupe élargi. C'est ce que fait le héros du Treizième guerrier, qui apprend la langue des Vikings, mais aussi le milliardaire Thomas Crown dans le film éponyme. Fils de paysan, Crown a intégré une grande université grâce au sport et commente ainsi son ascension sociale : «Les riches ne savent pas boxer. Apprendre à parler, ça a été le plus dur. » (avec un accent : «Rich kids 
cannae box. The hard part was learning to talk. »). Au sein d'un même pays, on parle des langues différentes, des sociolectes qu'il faut connaître pour intégrer le groupe : après avoir maîtrisé le langage du corps (ses aptitudes à la boxe lui valent une bourse), Crown a dû pour réussir apprendre la langue des puissants. L'extra-terrestre de Predator, lui, ne parvient jamais à établir la communication avec les humains car il imite leur langage de façon mécanique, comme un perroquet. La créature enregistre la voix de ses victimes humaines et se la repasse à plusieurs reprises. À la toute fin du film, Schwarzenegger pose au monstre la question de son identité («What the hell are you ?») mais le Prédator lui retourne sa question pour toute réponse. La créature est un miroir qui renvoie au personnage sa propre image et avec lequel tout dialogue est impossible.

À la poursuite d'Octobre rouge et Le Treizième guerrier nous montrent par contre comment le recours à une langue commune permet d'atténuer l'altérité. Dans le premier film, l'armée américaine cherche à détruire un sous-marin soviétique rebelle dont le commandant veut en réalité rejoindre les États-Unis : le passage d'une perception belliqueuse de la situation (les Russes menacent l'Amérique) à sa réalité pacifique se fait grâce à la pugnacité de Jack Ryan, un agent russophone de la CIA qui connaît et respecte la culture du sous-marinier au point d'être le seul à deviner ses intentions. Dans les premières scènes du Treizième guerrier, l'Autre est montré comme dégoûtant (les Vikings utilisent la même eau pour boire, se laver et cracher) et objet de railleries (les Vikings comparent le cheval d'ibn Fadhlan à un chien), avant qu'une langue partagée permette le passage de l'hostilité au respect puis à l'échange culturel. Le film représente trois stades d'évolution, trois niveaux de cultures : Ahmed ibn Fadhlan, le héros arabe, est un individu éclairé qui sait lire, écrire et parler plusieurs langues ; les Vikings, qui sont illettrés et ne connaissent qu'une langue, sont les représentants d'une culture en train de s'éteindre faute d'avoir su se développer; leurs adversaires wendols sont une anomalie de l'Histoire, une tribu préhistorique qui perpétue ses pratiques primitive avec des siècles de retard. Humains, les Wendols se considèrent comme des animaux (ils grognent, sont vêtus de peaux de bêtes, se terrent dans des grottes) et seront traités comme tels par les Vikings qui les chassent jusqu'à la mort. Toute communication entre les Wendols et la société humaine est impossible et leur rencontre anachronique ne peut aboutir qu'à l'élimination des premiers par les seconds. L'Arabe et les Vikings, par contre, se rapprochent par-delà leurs différences culturelles par le recours à une langue commune. La définition de la traduction par Paul Ricœur comme une «hospitalité langagière » (Ricœur, 2004, p. 19) trouve son application littérale dans la façon dont le héros de McTiernan est accueilli par une nouvelle communauté dès lors qu'il a appris à en parler la langue.

«Dans ...Octobre Rouge et Le Treizième guerrier, la distance entre les hommes était au cœur des films, et donc aussi leurs 
éloignements ou leurs rapprochements par le langage. » (McTiernan, 2003 , p. 24) À la fin d'À la poursuite d'Octobre rouge, des marins russes et américains font équipe pour gouverner un sous-marin en détresse, dans une métaphore politique transparente. McTiernan représente spatialement la distance qui se réduit entre les individus qui choisissent de parler la même langue. Les deux groupes, Américains et Soviétiques, se jaugent d'abord en silence, laissant entre eux une distance qui évoque un duel de western, gros plan sur un pistolet à l'appui. Le premier essai de communication ne se fait pas avec des mots mais par un geste d'une simplicité universelle : Ryan fait signe à un marin russe de lui donner une cigarette. Le rapprochement s'accentue lorsque les personnages s'aperçoivent qu'ils parlent les mêmes langues : l'Américain dit quelques mots en russe et le Soviétique lui répond en anglais. Cette inversion s'accompagne d'un changement dans l'échelle des plans, qui progresse vers le gros plan pour marquer une plus grande proximité entre les personnages. D'abord opposés frontalement par un champ/contre-champ, les peuples sont réunis dans la même image par un plan de profil où leurs deux représentants se serrent la main. Jack Ryan est placé à l'arrière-plan, dans l'axe de la poignée de main, faisant le lien entre les capitaines, entre les deux cultures, les deux langues : en tant qu'interprète, il est un intermédiaire.

Le Treizième guerrier développe cette figure de l'interprète. Envoyé en ambassade dans des terres lointaines, Ahmed ibn Fadhlan voyage en compagnie d'un interprète, Melchisider, qui l'aide à communiquer avec les Vikings. McTiernan décrit très précisément le processus d'interprétation, cette conversation triangulaire dont chaque phrase transite par un intermédiaire. Les cadrages soulignent le rôle de pivot de l'interprète : l'espace est organisé autour de la présence de Melchisider qui, dans un dialogue en champ/contre-champ entre ibn Fadhlan et le Viking Herger, apparaît au premier plan des deux côtés de l'image, faisant le lien visuel entre eux. L'interprétation de Melchisider s'adresse à son compagnon de route mais aussi au spectateur du film qui ne comprend pas non plus la langue des Vikings : McTiernan filme donc à plusieurs reprises le visage de l'interprète en gros plan et en regard caméra, tourné vers le public. Une fois posé ce processus d'interprétation, le réalisateur le simplifie: Melchisider passe hors-champ mais sa voix continue d'être présente sur la bande-son, par-dessus les dialogues incompréhensibles des Vikings dont il nous livre une interprétation simultanée.

Lorsqu'ibn Fadhlan devient son propre interprète, le personnage de Melchisider, devenu inutile, disparaît. Le héros apprend la langue des Vikings pour les comprendre et se faire comprendre d'eux, alors que, dans le roman dont le film est l'adaptation, il ne communiquait jamais directement avec ses compagnons, se contentant de parler en latin avec l'un d'entre eux, qui endossait à son tour la fonction d'interprète : 
Et moi, j'étais avec eux, incapable de parler leur langue ou de comprendre leur façon d'agir car mon interprète n'était pas venu avec nous. C'est seulement par le plus grand des hasards et par la grâce d'Allah que l'un de ces guerriers, Herger, se trouvait être un homme de talent qui savait un peu de latin. Avec son aide, je pus donc saisir la signification des évènements qui survinrent. (Crichton, 1976, p. 62)

Le romancier a pu contourner les lourdeurs du processus de traduction en éludant la répétition de chaque phrase en plusieurs langues, ce qui est plus difficile au cinéma. McTiernan choisit donc de faire apprendre à son personnage la langue des Vikings afin qu'il puisse communiquer directement avec eux. Concentré par le montage en une scène unique, cet apprentissage se fait grâce à un travail proprement cinématographique d'observation et d'écoute. "Les deux opération immédiatement observables que l'interprète effectue en même temps sont l'audition et l'énonciation. » (Lederer, 1981, p. 46) S'y ajoute pour le cinéma un travail d'observation : le personnage écoute mais McTiernan le filme en train de regarder, avec une série de gros plans sur ses yeux. Le passage des yeux d'ibn Fadhlan à la bouche des Vikings exprime visuellement le processus intellectuel de la traduction : il les regarde parler, les entend et les comprend. Le glissement d'une langue à l'autre se fait progressivement : au fur et à mesure que l'Arabe s'habitue à la langue de ses hôtes, les dialogues en langue originale (vieux norrois) sont remplacés par des répliques traduites (en anglais moderne). Il ne faut bien sûr pas croire que les Vikings se mettent soudainement à parler anglais pour communiquer avec un Arabe: McTiernan a choisi de faire parler ses personnages en anglais pour favoriser l'identification du spectateur américain. L'anglais est donc ici une langue mentale, par laquelle le spectateur perçoit ce qui est compris par le personnage principal : l'anglais remplace dans un premier temps l'arabe, puis la langue des Vikings, lorsque le héros a appris à la parler.

McTiernan n'impose pas l'anglais comme langue universelle, de façon ethnocentrique, il propose d'utiliser cet idiome unique pour remplacer la diversité des langues dans le cadre d'une fiction cinématographique. Dans À la poursuite d'Octobre rouge, le réalisateur effectue un zoom avant sur la bouche d'un personnage en train de parler russe ; lorsque la caméra se recule, sans coupure, il s'exprime désormais en anglais (ou dans la langue nationale de la version doublée) $)^{4}$.

Je me suis inspiré de Jugement à Nuremberg de Stanley Kramer [Judgment at Nuremberg, 1961] au cours duquel ils ont changé la langue comme une pure convention cinématographique: il est acquis que les gens parlent toujours allemand, mais nous n'allons plus nous embêter à le sous-titrer et à le lire. » (McTiernan, 2003, p. 24) 
Contrairement à l'apprentissage de la langue du Treizième guerrier, cette traduction n'est pas motivée par le récit : c'est un véritable coup de force du réalisateur qui, non content d'assumer la convention des personnages étrangers parlant anglais, souligne celle-ci par un effet de mise en scène. La scène illustre le remplacement arbitraire d'une langue par une autre, à des fins d'intelligibilité, qui est également le principe du doublage cinématographique. Le cinéaste se retrouve dans la position du traducteur, de celui qui choisit la langue qui sera entendue par le spectateur de son film. McTiernan est, de plus, confiant dans l'existence d'un «langage cinématographique » où ce sont moins les mots que les cadrages et les mouvements de caméra qui sont porteurs de sens. Dans ce cas, la question de la traduction ne se pose plus seulement d'une langue à une autre mais d'un langage à un autre, de la parole à l'image.

\section{L'image, un langage universel ?}

John McTiernan met son spectateur en position d'apprécier la valeur des cultures et des langues étrangères par le biais de la traduction ( $\grave{A} l a$ poursuite d'Octobre rouge) et de l'immersion anthropologique (Le Treizième guerrier). Le cinéaste fait pourtant peu de cas des dialogues, qu'il présente comme un élément parmi d'autres de la bande-son :

Je conçois le cinéma comme de l'opéra, les mots eux-mêmes n'ont aucune espèce d'importance, c'est la façon dont ils sont dits qui prime, de quoi ont l'air ceux qui les prononcent au moment où ils les prononcent. Les mots ne sont qu'un bruit, ce ne sont pas eux qui font avancer l'histoire. (McTiernan, 2003, p. 24)

Une telle assertion renvoie à Alfred Hitchcock déclarant, à propos de Fenêtre sur cour [Rear Window, 1954], que «le dialogue doit être un bruit parmi les autres, un bruit qui sort de la bouche des personnages dont les actions et les regards racontent une histoire visuelle » (Hitchcock, 1966, p. 183), mais c'est surtout le dramaturge Antonin Artaud que McTiernan se plaît à citer (McTiernan, 1986, p. 18 ; 1995, p. 40). Pour Artaud le langage purement théâtral,

s'il existe, se confond nécessairement avec la mise en scène considérée :

$1^{\circ}$ D'une part, comme la matérialisation visuelle et plastique de la parole.

$2^{\circ}$ Comme le langage de tout ce qui peut se dire et se signifier sur une scène indépendamment de la parole, de tout ce qui trouve son expression dans l'espace, ou qui peut être atteint ou désagrégé par lui. (Artaud, 1964, p. 106) 
C'est un «langage par signes, par gestes et attitudes ayant une valeur iconographique tels qu'ils existent dans certaines pantomimes non perverties. » (p.59)

Pour McTiernan, la parole n'a pas ou peu de valeur informative et il demande à son spectateur d'être attentif non pas au contenu du discours mais à la façon dont il est prononcé et, surtout, filmé. Une scène de dialogue de Thomas Crown, en roumain non sous-titré, illustre cette posture. L'enquêteuse Catherine Banning interroge dans sa langue un cambrioleur qui ne parle pas anglais. McTiernan avait d'abord pensé sous-titrer la scène avant d'y renoncer, car cela aurait poussé la plupart des spectateurs à lire les sous-titres au lieu d'observer l'attitude et les déplacements de Banning, qui suffisent à transmettre le sens de la scène (Commentaire audio du DVD MGM, 31 minutes 50 secondes). L'ajout de mentions écrites est hétérogène avec la dimension figurative de l'image cinématographique. De plus, la traduction verbale (doublage) ou écrite (sous-titres) apparaissent comme une facilité au regard des possibilités expressives du médium. Alors que la compréhension de dialogues en langue étrangère nécessite de la part du spectateur des compétences extérieures à celles du visionnage cinématographique, McTiernan filme de façon à faire porter le sens de la scène par la seule image. Pour compenser la non-intelligibilité des dialogues, il met m'accent sur les mouvements ondoyants du personnage, qui enveloppe le suspect, comme un animal sauvage sa proie. Le resserrement du cadre mime le piège qui se referme sur le criminel. Il ne s'agit pas seulement d'une pantomime, où le corps l'emporte sur la parole, mais de la recherche d'une expressivité propre à la caméra. Le sens des mots est renforcé par une mise en scène basée sur le mouvement des corps dans l'espace et leur relation à l'appareil de prise de vue.

La volonté du cinéaste de filmer la parole comme un phénomène physique est évidente dès les scènes de conversation téléphoniques de Piège de cristal, et trouve son aboutissement dans Basic. Ce dernier film n'est pas un film d'action mais un whodunit composé de longues scènes de discussion où les dialogues, pourtant en anglais, semblent optionnels : le réalisateur choisit ainsi de rendre inaudible une des révélations finales, masquant les paroles par un bruit assourdissant. Les personnages bougent leurs lèvres en silence mais leur posture suffit à faire comprendre au spectateur l'importance de la situation. Tout le monde ment dans Basic: les dialogues sont trompeurs et McTiernan demande à son spectateur de se fier plutôt aux images. Tout au long du film, les affrontements psychologiques et verbaux sont traités comme autant de luttes physiques, durant lesquelles les personnages se déplacent de façon chorégraphique. La caméra suit le mouvement de la parole, le cheminement des mots des idées à travers l'espace.

Le théoricien Jean Mitry voyait dans le cinéma «un moyen d'expression [qui], si l'on s'en tient au sens restrictif du mot, ne permet de traduire que des sentiments, des émotions : il ne saurait exprimer des 
idées. » [Mitry, 1963, p. 29] Le travail de John McTiernan est pourtant bien de donner un équivalent visuel aux processus intellectuels de ses personnages, comme la manipulation psychologique (Thomas Crown), l'induction logique (d'À la poursuite d'Octobre rouge, Basic) ou l'apprentissage d'une langue étrangère (Le Treizième Guerrier). McTiernan agence ses images de façon à amener le spectateur à comprendre la langue de l'autre. Nous ne sommes pas ici en présence d'une «traduction interlinguale ou traduction proprement dite [qui] consiste en l'interprétation des signes linguistiques au moyen d'une autre langue », mais plutôt d'une «traduction intersémiotique ou transmutation [qui] consiste en l'interprétation des signes linguistiques au moyen de systèmes de signes non linguistiques » (Jakobson, 1963, p. 79). La parole est « traduite » en images cinématographiques. Il y a en somme pour John McTiernan deux modes d'expression et de connaissance, l'un verbal et l'autre non, le premier lié aux dialogues et le second aux images. Le travail du cinéaste serait alors de traduire la parole en images, de remplacer un énoncé linguistique par une création plastique de sens équivalent.

La diversité des langues est source de confusion, alors que l'image, unique, serait compréhensible par tous et partout. Cette croyance en une "pureté » de l'expression cinématographique a été critiquée, avant même que McTiernan ne commence à faire des films, mais métrite d'être interrogée à nouveau. Ainsi, pour Jean Mitry,

il est évident que le cinéma ne saurait être un langage si l'on s'en tient à la définition classique qui veut que le langage n'utilise que des signes abstraits de forme fixe et de signification constante. (Mitry, 1963, p. 32) Il ne saurait donc y avoir de grammaire du film pour l'extrême raison que toute grammaire se fonde sur la fixité, l'unité et la conventionalité des signes. Elle ne peut régir que des modalités se référant à cette fixité fondamentale. (p. 502)

Christian Metz critique lui aussi le principe du «langage cinématographique » comme grammaire cohérente. Il observe plutôt « un système singulier, propre à un seul film; au fond, ce n'est plus vraiment du langage cinématographique qu'il s'agit, mais de l'emploi qui en est fait dans un cas précis. » (Metz, 1977, p. 50) McTiernan a pourtant élaboré, pour transcrire visuellement des processus intellectuels, un système visuel repris de films en films : il place des sources lumineuses brillantes derrière un personnage en train d'avoir une idée - comparable au pictogramme de l'ampoule en bande dessinée - et fait passer une portion de l'image du flou au net pour marquer la transmission de la pensée à travers l'espace, d'un personnage à un autre. Ces effets caractérisent par leur récurrence le style du réalisateur mais leur absence à peu près partout ailleurs au cinéma montre bien, à la suite de Metz, qu'il ne s'agit pas d'un langage organisé, accepté et partagé par tous. 
McTiernan aime à comparer l'expressivité non-verbale de sa mise en scène à celle d'un air d'opéra, par opposition à la contingence des mots du scénario ou du livret. Les images et les sons ne sont pourtant pas plus universels que les mots et il faut apprendre voir autant qu'à lire et écouter. Pour comprendre un film, avec ou sans dialogues, il faut en maîtriser la «langue », les codes visuels qui, à partir de conventions communes, varient en fonction du lieu et de l'époque (les films de McTiernan sont à ce titre de purs produits du cinéma hollywoodien des années 1980-90, avec leurs mouvements de caméra rapides, leur éclairage irréaliste et, dans Rollerball, l'influence esthétique du clip vidéo).

Pour Christian Metz, le cinéma repose sur des «configurations mixtes » (Metz, 1977, p. 25) d'où la parole et plus largement l'expression verbale ne sont pas exclues, bien au contraire: «le discours cinématographique inscrit ses configurations signifiantes dans des supports sensoriels de cinq ordres: l'image, le son musical, le son phonétique des "paroles", le bruit, le tracé graphique des mentions écrites. » (p. 10) Malgré l'importance donnée aux images, le cinéma ne peut donc pas se passer des mots :

Chaque film est le lieu d'un rendez-vous productif (plus ou moins productif) entre le cinéma et ce qui n'est pas lui. (p. 83)

Il est mille choses dans un film, qui ne viennent pas du cinéma (même si leur mise en œuvre au sein du film - leur « traitement »est susceptible d'emprunter des voies proprement cinématographiques): ainsi de tout ce matériel filmique communément recensé sous des étiquettes comme «psychologie des personnages », «étude de mœurs », « arrière-fond psychanalytique », «thèse sociale » du film (ou religieuse, ou politique), «thématique », etc. (p. 54)

Ce n'est pas parce qu'un message est visuel que tous ses codes le sont ; ce n'est parce qu'un code se manifeste dans des messages visuels qu'il ne se manifeste pas aussi ailleurs. Les «langages » visuels entretiennent avec les autres des liens systématiques qui sont multiples et complexes, et on ne gagne rien à opposer le « verbal » et le « visuel ». (p. 24)

Il est ainsi impossible de comprendre réellement le face à face entre Américains et Soviétiques d'À la poursuite d'Octobre rouge sans connaitre un tant soit peu la situation de la Guerre froide (savoir extracinématographique), quand bien même les cadrages de McTiernan spatialisent la relation entre les deux cultures sans recourir à la moindre explication verbale. Les «configurations mixtes» de Metz renvoient à une autre forme de multilinguisme : non pas plusieurs langues cohabitant sur la bande-son, mais un dialogue productif entre ces différents langages que seraient les différentes formes artistiques à l'œuvre dans la création cinématographique, du cadrage au montage, du son aux mouvements de 
la caméra. La traduction consiste à trouver une équivalence entre les mots et les expressions d'une langue dans une autre, à « dire presque la même chose » (Eco, 2003). En littérature, la traduction vient remplacer le texte original, alors que dans un film sous-titré deux langues coexistent, l'une sur la bande-son et l'autre à l'image. Voir un film en maîtrisant plusieurs des codes esthétiques qui le composent revient alors en quelque sorte à lire un livre bilingue (ou plus) : le spectateur-lecteur est en présence de plusieurs textes (un complexe audio-visuel et narratif) parallèles qui se complètent en racontant «presque » la même chose; semblables mais différents.

\section{Conclusion}

Les affrontements interculturels dont regorgent les films d'action américains témoignent, consciemment ou non, une forme de nationalisme. La supériorité des héros de John McTiernan dans de tels combats n'est pas forcément physique mais toujours intellectuelle : pour vaincre l'autre, il faut le comprendre et le prendre à son propre piège (Piège de cristal). Un autre aspect des œuvres du réalisateur est tout aussi important : au lieu de combattre l'altérité, le héros s'allie avec elle dans un respect mutuel (À la poursuite d'Octobre rouge, Le Treizième guerrier). La traduction interlinguale apparait alors comme le moyen pour des individus de langues et de cultures différentes de communiquer entre eux, par le biais d'un traducteur. Le besoin d'un tel intermédiaire disparait vite puisque McTiernan confie à sa mise en scène (cadrage, déplacement de la caméra, lumière, montage...) le soin de prendre en charge le passage d'une langue à l'autre. On est alors dans le cadre d'une traduction intersémiotique où le texte verbal se voit doté d'un équivalent visuel. La possibilité théorique d'un langage "purement» visuel a été âprement discuté mais, au même titre que celle d'Alfred Hitchcock, l'œuvre de John McTiernan tente de la mettre en pratique. Le dynamisme spectaculaire du cinéma d'action hollywoodien n'est plus alors une fin en soi mais est utilisé comme un mode d'expression au travers duquel sont traduites non seulement les paroles mais aussi les pensées des personnages.

\section{Références}

Amiel, V., \& Couté, P. (2003). Formes et obsessions du cinéma américain contemporain. Paris : Klincksieck.

Artaud, A. (2003). Le théâtre et son double. Paris : Gallimard, Folio essais. (Ouvrage original publié en 1964) 
Crichton, M. (1999). Les Mangeurs de morts (L. Rosenbaum, trad.). Paris : Pocket. (Ouvrage original publié en 1976 sous le titre Eaters of the Dead. New York, NY : Knopf)

Cronin, M. (2008). Translation goes to the Movies. Londres et New York : Routledge. Eco, U. (2007). Dire presque la même chose: expériences de la traduction (M. Bouzaher, trad.). Paris : Grasset. (Ouvrage original publié en 2003 sous le titre Dire quasi la stessa cosa, esperienze di traduzione. Milan: Bompiani).

Hoberman, J. (2009). Notre Arnold, le surdéterminator. Dans J. Hoberman, The magic hour : une fin de siècle au cinéma (M.-M. Burdeau, trad.) (pp. 47-60). Nantes : Capricci. (Ouvrage original publié en 2003 sous le titre The Magic Hour: Film at Fin De Siecle. Culture and the Moving Image. Philadelphia, PA: Temple University Press].

Jakobson, R. (1963). Aspects linguistiques de la traduction. Dans R. Jokobson, Essais de linguistique générale tome 1. (N. Ruwet, Trad.) (pp. 78-86). Paris : Les Éditions de Minuit. (Ouvrage original publié en 1959 sous le titre On linguistic Aspects of Translation. Cambridge, MA: Harvard University Press).

Lederer, M. (1981). La traduction simultanée : expérience et théorie. Paris : Lettres modernes.

Lévi-Strauss, C. (1962). La Pensée sauvage. Paris : Plon.

McTiernan, J. (1986, juin). Nomads Land (entretien avec Chenu, G.). Starfix, 37, 1718.

McTiernan, J. (1995, juillet-août). Entretien avec P. Graves. Le Cinéphage, 21, 40.

McTiernan, J. (2003, mars). Dans la jungle (entretien avec C. Cohen, O. Joyard et J.-M. Lalanne). Cahiers du cinéma, 577, 18-25.

Metz, C. (1977). Langage et cinéma. Paris : Éditions Albatros.

Mitry, J. (1963, 2001). Esthétique et psychologie du cinéma. Paris : Éditions du Cerf.

Ricœur, P. (2004). Sur la traduction. Paris : Bayard.

Truffaut, F., Scott, H. (1966, 1993). Hitchcock. Paris: Gallimard.

Valantin, J.-M. (2010). Hollywood, le Pentagone et le monde : les trois acteurs d'une stratégie mondiale. Paris : Autrement.

Vincendeau, G. (2009). Acteurs européens et cinéma classique hollywoodien Casablanca, accents et authenticité. Dans J. Nacache \& J.-L. Bourget (dirs.), Le classicisme hollywoodien (pp. 189-202). Rennes : Presses Universitaires de Rennes.

\section{Filmographie}

Elwes, C., Kastner, E., Pappas, G. (Producers), \& McTiernan, J. (Director). (1986). Nomads [Motion picture]. United States of America : Cinema VII, Producers Sales Organization.

Davis, J., Gordon, L., Silver, J. (Producers), \& McTiernan, J. (Director). (1987). Predator [Motion picture]. United States of America : Amercent Films, American Entertainment Partners L.P., Davis Entertainment, Lawrence Gordon Productions, Silver Pictures, Twentieth Century Fox Film Corporation.

Gordon, L., Silver, J. (Producers), \& McTiernan, J. (Director). (1987). Die Hard [Motion picture]. United States of America : Twentieth Century Fox, Gordon Company, Silver Pictures. 
Neufeld, M. (Producer), \& McTiernan, J. (Director). (1990). The Hunt for Red October [Motion picture]. United States of America : Paramount Pictures, Mace Neufeld Productions, Nina Saxon Film Design.

Dubrow, D., Vajna, A. G. (Producers), \& McTiernan, J. (Director). (1992). Medicine Man [Motion picture]. United States of America : Cinergi Pictures Entertainment, Hollywood Pictures.

McTiernan, J., Roth, S. (Producers], \& McTiernan, J. (Director). (1993). Last Action Hero [Motion picture]. United States of America : Columbia Picture, Oak Productions.

McTiernan, J., Tadross, M. (Producers), \& McTiernan, J. (Director). (1995). Die Hard: With a Vengeance [Motion picture]. United States of America : Cinergi Pictures Entertainment, Twentieth Century Fox Film Corporation.

Crichton, M., Dowd, N., McTiernan, J. (Producers), \& McTiernan, J. (Director). (1995). The 13th Warrior [Motion picture]. United States of America : Touchstone Pictures.

Brosnan, P., Saint-Clair, B. (Producers), \& McTiernan, J. (Director). (1999). The Thomas Crown Affair [Motion picture]. United States of America : United Artists, Irish DreamTime, Metro-Goldwyn-Mayer Pictures.

McTiernan, J., Roven, C., Saint-Clair, B. (Producers), \& McTiernan, J. (Director). (2002). Rollerball [Motion picture]. United States of America, Germany, Japan : Atlas Entertainment, Helkon Media AG, Metro-Goldwyn-Mayer, Mosaic Media Group, Toho-Towa, Yorktown Productions

Medavoy, M., Messer, A., Tadross, M., Vanderbilt, J. (Producers), \& McTiernan, J. (Director). (2003). Basic [Motion picture]. United States of America, Germany : Phoenix Pictures, Intermedia Films.

1 L'Australien Mel Gibson (L'Arme fatale [Lethal Weapon, Richard Donner, 1987] est au moins originaire d'un pays anglophone.

2 Pour les références des films de John McTiernan, se reporter à la filmographie en fin de texte.

3 La version allemande de Piège de cristal masque l'origine germanique des terroristes en les affublant de patronymes anglais.

4 Le générique du film propose un semblable glissement : le titre écrit en cyrillique se change en alphabet latin. 